Surveys in Differential Geometry XV

\title{
Automorphisms of Graded Super Symplectic Manifolds
}

\author{
Joshua Leslie
}

\begin{abstract}
In section 1 we present the language of differential analysis in graded locally convex infinite dimensional vector spaces that was studied in [5].

In section 2 we give results on diffeological Lie groups and Lie algebras and formulate diffeological versions of the fundamental theorems of Lie.

In section 3 we introduce the notion of graded supermanifolds and we indicate a method of constructing non-trivial compact graded supermanifolds with non-degenerate graded differential forms which generalize symplectic and contact structures to the graded case.

In section 4 we study automorphisms of generalizations of some of the classical geometric structures to compact graded supermanifold, $M$, and show that these automorphism groups are diffeological Lie subgroups of the Lie group of superdiffeomorphisms of $M$.
\end{abstract}

\section{Introduction}

We shall show how to construct compact supermanifolds having geometric structures determined by certain elements of

$$
\Gamma\left(\wedge^{p}\left(T_{\Gamma}^{\star} M\right) \otimes_{\Gamma_{0}} \otimes_{\Gamma_{0}}^{q}\left(T_{\Gamma} M\right)\right)
$$

where $\Gamma\left(\wedge^{p}\left(T_{\Gamma}^{\star} M\right) \otimes_{\Gamma_{0}} \otimes_{\Gamma_{0}}^{q}\left(T_{\Gamma} M\right)\right)$ represents the smooth sections of the bundle

$$
\Pi_{M}: \wedge^{p}\left(T_{\Gamma}^{\star} M\right) \otimes_{\Gamma_{0}} \otimes_{\Gamma_{0}}^{q}\left(T_{\Gamma} M\right) \mapsto M,
$$

where by abuse of notation the " $\Gamma$ " in $T_{\Gamma}^{\star} M$ and $T_{\Gamma} M$ represents a finitely generated Grassmann algebra. The geometric automorphisms of these geometric structures will be seen to be diffeological Lie subgroups of the infinite

(C)2011 International Press 
dimensional Lie group of $G^{\infty}$ diffeomorphisms of an a non trivial compact $G^{\infty}$ supermanifold.

Underlying the infinite dimensional Lie algebras of Cartan are geometric structures of differential geometry; such as contact structures, symplectic structures, and foliated structures. It is known [4] in the the compact case that the automorphism groups of these geometric structures are Lie subgroups of the Lie group of diffeomorphisms of the underlying differentiable manifold. We show this is also the case for the generalized contact and symplectic structures on graded $G^{\infty}$ super manifolds.

\section{Analysis in graded infinite dimensional topological vector spaces}

We designate by $N$ the natural numbers. Let $\Gamma$ be the $N$ graded Grassmann algebra over the reals, $R$, of super numbers generated by an arbitrary set $\mathcal{X}=\left\{\xi_{i}\right\}_{i \in I}, \operatorname{deg}\left(\xi_{i}\right)=1$ ), when $\mathcal{X}$ is infinite we suppose that $\Gamma$ is furnished with the topology given by the inductive limit of $\Gamma_{K}$, for $K \in J$, where $J$ is the collection of finite subsets of $I$ ordered by inclusion and $\Gamma_{K}$ the finite dimensional subspaces of $\Gamma$ generated by $\xi_{i_{1}}, \ldots, \xi_{i_{n_{K}}}$. With this topology $\Gamma$ is a complete locally convex topological vector space. $\Gamma$ is a $Z_{2}$ graded commuatative (i.e. $a b=(-1)^{|a||b|} b a$ algebra, where $|a|$ designates the parity of $a$. $\Gamma$ with this topology will be used as our base ring when $\mathcal{X}$ is infinite. Note that $\Gamma$ is a bigraded $\left(\left(N, Z_{2}\right)\right)$ ring, graded over the non-negative integers $N$ by the the number of generators, $\xi \in X$ in the expression of an element and by the $Z_{2}$-parity of that number. When we consider $\Gamma$ as a right $\Gamma$ module there are two possible right $\Gamma$ module structures potentially of interest for us.

Let $\epsilon \Gamma: \Gamma \mapsto R$ be the unique $R$ - algebra homomorphism such that $\epsilon_{\Gamma}\left(k 1_{\Gamma}\right)=k, k \in R$, and set $I(\Gamma)=k e r(\epsilon)$, we have $\Gamma=K \dot{1}_{\Gamma}+I(\Gamma)$.

Define a right $\Gamma$ module structure on $\Gamma$ noted $\Gamma_{R}$ by $(k, \gamma) \dot{\gamma}^{\prime}=(-1)^{|\gamma|\left|\gamma^{\prime}\right|}$ $\left.k \dot{\epsilon}\left(\gamma^{\prime}\right), \gamma \dot{\gamma}^{\prime}\right)$, where $k \in K, \gamma \in I(\Gamma)$, and $\gamma^{\prime} \in \Gamma$. Note that with this right $\Gamma$ module structure $\Gamma_{R}$ remains a right bigraded $\Gamma$ module. When the right $\Gamma$ module structure on $\Gamma$ is given by right $\Gamma$ multiplication by abuse of notation we shall simply designate by $\Gamma$ this right $\Gamma$ module. Let $\Gamma_{0}\left(\operatorname{resp} . \Gamma_{1}\right)$ be the even (resp. odd) $\Gamma_{0}$-submodule of $\Gamma$.

Let $V$ and $W$ be topological graded modules over $\Gamma_{0}$, a continuous mapping $f: V \times \cdots \times V \mapsto W$ is said to be an $n$-multimorphism when $f$ is n-multilinear with repect to the ground field $K$ and

$$
f\left(e_{1}, \ldots, e_{i} \gamma, e_{i+1}, \ldots, e_{n}\right)=f\left(e_{1}, \ldots, e_{i}, \gamma e_{i+1}, \ldots, e_{n}\right), \quad \gamma \in \Gamma_{0}
$$

and

$$
f\left(e_{1}, \ldots, e_{n} \gamma\right)=f\left(e_{1}, \ldots, e_{n}\right) \gamma, \quad \gamma \in \Gamma_{0} .
$$

In case $\Gamma$ is finite dimensional a $\Gamma$ - multimorphism is called regular when there exists a countably generated infinite dimensional algebra $\overline{\Lambda \text { containing }}$ 
$\Gamma$ as a proper unitary subalgebra such that the map

$$
f_{\Lambda_{0}}: V_{\Lambda_{0}} \times \cdots \times V_{\Lambda_{0}} \mapsto W_{\Lambda_{0}}
$$

is a $\Lambda_{0^{-}}$multimorphism extension of $f$, where $V_{\Lambda_{0}}=V \bigotimes_{\Gamma_{0}} \Lambda_{0}$, defined by $f_{\Lambda_{0}}\left(v_{1} \otimes \lambda_{1}, \ldots, v_{n} \otimes \lambda_{n}\right)=f\left(v_{1}, \ldots, v_{n}\right) \otimes \lambda_{1} \ldots \lambda_{n}$.

Definition 1.1. Let $V$ and $W$ be topological graded modules over $\Gamma_{0}$, given $U \subseteq V$ open, a function $f: U \mapsto W$ will be called super smooth when for every $n \geq 1$ there exists continuous maps, which are regular $k$-multimorphisms in the $k$-terminal variables, for $x \in U$ fixed: $D^{k} f(x ; \ldots)$ : $U \times V \times \cdots \times V \mapsto W, k \leq n$, such that

$F_{k}(h)=f(x+h)-f(x)-\frac{1}{1 !} D f(x, h)-\cdots-\frac{1}{k !} D^{k} f(x, h, \ldots, h), 1 \leq k \leq n$,

satisfies the property that

$$
G_{k}(t, h)=\left\{\begin{array}{cc}
F_{k}(t h) / t^{k}, & t \neq 0 \\
0, & t=0
\end{array}\right.
$$

is continuous at $(0, \mathrm{~h}) \forall h \in V$.

Proposition 1.1. If a function $f: U \mapsto W$ is smooth and $D f(x ; \alpha \gamma)=$ $D f(x ; \alpha) \gamma$, then $f(x)$ is super smooth.

Proof. In this proof we suppose that $D^{k} f\left(x ; \alpha_{1}, \ldots \alpha_{k},\right): U \times V \times \cdots \times$ $V \mapsto W$ symmetric in $\alpha_{1}, \ldots \alpha_{k}$ It suffices to prove for $n \geq 2$ that a $C^{n}$ function $f: U \mapsto W$ which is $G^{n-1}$ is $G^{n}$. It is known that $D_{t=0} D^{k} f(x+$ $t h, h \ldots, h)=D^{k+1} f(x+t h, h \ldots, h)$. For $k \geq 2$, we have $D^{k} f(x+t h, h \ldots$, $h \gamma)=D_{t=0}\left(D^{k-1} f(x+t h, h \ldots, h) \gamma\right)=D_{t=0} D^{k-1} f(x+t h, h \ldots, h) \gamma=D^{k}$ $f(x+t h, h \ldots, h) \gamma$.

Super smooth functions are smooth and therefore satisfy the classical theorems such as the chain rule.

Note that multiplication defines a super smooth map

$$
\Gamma \times \Gamma \mapsto \Gamma .
$$

Given a well-ordering, $\prec$, of $I$, we obtain an associated basis of $\Gamma,\left\{\xi_{i_{1}}, \ldots\right.$, $\left.\xi_{i_{n}}\right\}$ of $\Gamma$, where $i_{k} \prec i_{k+1}$; we shall call this basis a canonical basis of $\Gamma$.

To each of these basis elements is associated an unique $1-$ morphism $p_{\left\{\xi_{i_{1}}, \ldots, \xi_{i_{n}}\right\}}: \Gamma \mapsto \Gamma$, defined by

$$
\begin{aligned}
& p_{\left\{\xi_{i_{1}} \ldots \xi_{i_{n}}\right\}}\left(a_{\left.j_{1} \ldots j_{n_{1}} \xi_{i_{1}} \ldots \xi_{j_{n_{1}}}+\cdots+a_{l_{1} \ldots l_{n}} \xi_{l_{1} \ldots \xi_{l_{n}}+\cdots+a_{k_{1} \ldots k_{n}}} h \xi_{k_{1}} \ldots \xi_{k_{n_{2}}}\right)}\right) \\
& \quad=a_{i_{1}, \ldots, i_{n}} \xi_{i_{1}}, \ldots, \xi_{i_{n}}
\end{aligned}
$$

It is straightforward to verify that as in the classical case

Proposition 1.2. If $f: V \mapsto W$ is a continuous $\Gamma_{0}$ homomorphism, then $f(x)$ is a supersmooth function such that $D_{x} f=f \forall x \in V$. 
Given an open subset $\mathrm{U}$ of $\Gamma . x_{0} \in U, C^{\infty}(U, \Gamma)$ has the structure of a graded module over the ground field $K$ determined by $C^{\infty}(U, \Gamma)_{0}=C^{\infty}(U$, $\left.\Gamma_{0}\right)$ and $C^{\infty}(U, \Gamma)_{1}=C^{\infty}\left(U, \Gamma_{1}\right)$.

Designate by $\Gamma_{0}^{n}$ (resp. $\Gamma_{1}^{n}$ ) the n-fold direct sum of the $\Gamma_{0}$ modules $\Gamma_{0}$ (resp. $\Gamma_{1}$ ) and suppose $U \subset \Gamma_{0}^{n} \oplus \Gamma_{1}^{m}$ is a non-empty open subset, set $\eta_{i}=\left(0, \ldots, 0,1_{\Gamma}, 0, \ldots, 0\right) \in \Gamma_{0}^{n} \oplus \Gamma_{1}^{m}$, where $1_{\Gamma}$ is in the ith position, $i \leq n$. Given a smooth function $f: U \mapsto \Gamma$ set $\partial f / \partial x_{i}(x)=D_{t=o} f\left(x+t \eta_{i}\right)$.

Given a system of generators $\left\{\xi_{i}\right\}$ of $\Gamma$ define a linear map $\Delta_{\xi_{i}}: \Gamma \mapsto$ $\Gamma$ by $\Delta_{\xi_{i}}\left(\xi_{i_{1}} \ldots \xi_{k} \ldots \xi_{i_{n}}\right)=\sum_{k=1}^{n} \delta_{i i_{k}}(-1)^{(k-1)}\left(\xi_{i_{1}} \ldots \xi_{i_{k-1}} \xi_{i_{k+1}} \ldots \xi_{i_{n}}\right)$, and $\Delta_{\xi_{j}}\left(1_{\Gamma}\right)=0$.

Lemma 1.1. $\Delta_{\xi_{i}}: \Gamma \mapsto \Gamma$ is a 1-morphism, and a graded odd derivation; that is, $\Delta_{\xi_{i}}\left(\gamma_{1} \cdot \gamma_{2}\right)=\Delta_{\xi_{i}}\left(\gamma_{1}\right) \cdot \gamma_{2}+(-1)^{\left|\gamma_{1}\right|} \gamma_{1} \cdot \Delta_{\xi_{i}}\left(\gamma_{2}\right)$, " $|\cdot|$ " designates "the parity of" (0 for even, 1 for odd). $\Delta_{\xi_{i}}$ is odd in the sense that $\Delta_{\xi_{i}}\left(\Gamma_{i}\right) \subset \Gamma_{i+1}$.

Proof. By definition $\Delta_{\xi_{i}}$ is a 1-morphism. To see that $\Delta_{\xi_{i}}$ is a derivation it suffices to consider $\Delta_{\xi_{i}}\left(\gamma_{1} \cdot \gamma_{2}\right)$ and to do an induction on the Z-grading of $\gamma_{1}$.

Definition 1.2. Let $X \subset \Gamma$ be a set of generators of the Grassmanian algebra $\Gamma$. given an open subset $\mathrm{U}$ of $\Gamma$. $x_{0} \in U$, a generator $\xi \in X \subset \Gamma$, and a smooth function $f: U \mapsto \Gamma$ set $\partial f / \partial \xi(x)=\Delta_{\xi} \circ D_{t=0} f(x+t \xi)$.

We have the following important proposition which is immediate from the definitions:

Proposition 1.3. Let $x \in U, \quad \partial f / \partial \xi(x): C^{\infty}\left(U, \Gamma_{i}\right) \mapsto C^{\infty}\left(U, \Gamma_{i+1}\right)$ defines a smooth odd derivation for the $C^{\infty}$ topology.

Designate by $\mathcal{L}_{n}(\Gamma, \Gamma)$ the vector space of n-multilinear n-morphisms.

Proposition 1.4. Suppose that in $\mathcal{L}_{1}(\Gamma, \Gamma)$ we take set theoretic composition as a product. Then $\mathcal{L}_{1}(\Gamma, \Gamma)$ is a $Z_{2}$-graded algebra, where $\mathcal{L}_{1}(\Gamma, \Gamma)_{0}=$ $\left\{T \in \mathcal{L}_{1}(\Gamma, \Gamma): T\left(\Gamma_{0}\right) \subset \Gamma_{0}\right.$ and $\left.T\left(\Gamma_{1}\right) \subset \Gamma_{1}\right\}$, and $\mathcal{L}_{1}(\Gamma, \Gamma)_{1}=\left\{T \in \mathcal{L}_{1}(\Gamma, \Gamma):\right.$ $T\left(\Gamma_{0}\right) \subset \Gamma_{1}$ and $\left.T\left(\Gamma_{1}\right) \subset \Gamma_{0}\right\}$.

Proposition 1.5. Given an open subset $U$ of $\Gamma, x_{0} \in U$, a generator $\xi \in X \subset \Gamma$, and a super smooth function $f: U \mapsto R \subset \Gamma$. Then $\partial f / \partial \xi(x)=0$.

Proof. Recall that $D f(x,$.$) extends to countably infinite generated \Lambda$ where $\Gamma \subset \Lambda . \partial f / \partial \xi(x) \neq 0$. The real number $D_{t=0} f(x+t \xi)$ satisfies for every element $\eta \in \Lambda$ that $0=D f(x ; \xi \xi \eta)=D f(x ; \xi) \xi \eta$. Therefore, $\partial f / \partial \xi(x)=\Delta_{\xi} \circ$ $D_{t=0} f(x+t \xi)=0$.

Definition 1.3. Given an open subset $U \subset \Gamma_{0}^{n} \oplus \Gamma_{1}^{m}$, a graded derivation $D=D_{0}+D_{1}: G^{\infty}(U, \Gamma) \mapsto G^{\infty}(U, \Gamma)$, is a $\Gamma$ homomorphism, where $\Gamma$ is acting from the right, such that $D_{0}: G^{\infty}(U, \Gamma) \mapsto G^{\infty}(U, \Gamma)$ is a derivation, and $D_{1}: G^{\infty}\left(U, \Gamma_{i}\right) \mapsto G^{\infty}(U, \Gamma i+1)$ satisfies $D_{1}(f \cdot g)=D_{1}(f) \cdot g+(-1)^{|f|} f$. $D_{1}(g)$, where $|f|$ designates the parity of $\mathrm{f}$. 
Using the fact that a $G^{\infty}$ super smooth functions $f: U \mapsto \Gamma$ is a $C^{\infty}$ smooth function we deduce from the Taylor series with Lagrange remainder that

Proposition 1.6. Every smooth derivation $D: G^{\infty}(U, \Gamma) \mapsto G^{\infty}(U, \Gamma)$ is of the form $D=\sum_{i=1}^{n} g_{i}(x) \partial / \partial \xi_{i}+\sum_{j=1}^{N} f_{j}(x) \partial / \partial x_{j}$, where $f_{j}, g_{i} \in C^{\infty}$ $(U, \Gamma)$.

\section{Diffeological lie groups and lie algebras}

The details for this section can be found in [7]. Given the category $\mathcal{M}$ whose objects are manifolds modelled on the open subsets of complete Hausdorff locally convex topological vector spaces, and whose morphisms are $C^{\infty}$ functions. A diffeological space is a set $S$ together with a contravariant subfunctor on $\mathcal{M} F_{S}(N) \subseteq H_{\text {om }}(N, S)$ such that constant maps are in $F(N)$ for each object $N$ of $\mathcal{M}$ and each $x \in S$ and such that $F$ restricted to the subcategory of open subsets of a fixed manifold, $N$, whose morphisms are the canonical injection of open subsets of $N$ into each other, $F$ satisfies the axioms of a set valued sheaf. Given an open $U \subset E, E$ a graded complete Hausdorff locally convex topological vector space. An element $f \in F_{S}(U)$ is called a plot of $S$.

Given diffeological spaces $\left(X_{1}, F_{1}\right)$ and $\left(X_{2}, F_{2}\right)$ a function $f: X_{1} \mapsto X_{2}$ is called smooth or $C^{\infty}$ if for each $g \in F_{1}$, we have $f \circ g \in F_{2}$. We shall say that $f$ is locally smooth at $x_{0} \in X_{1}$ when given any smooth map, $g \in F_{1}$, from a neighborhood, $U$, of $0 \in E$, where $f(0)=x_{0}$ there exists a neighborhood, $U_{0} \subset U$ of 0 such that $f \circ\left(g \mid U_{0}\right) \in F_{2}$.

When $S$ is a $C^{\infty}$ manifold we shall suppose without explicit mention to the contrary that it has its underlying diffeology given by $F(C)$ being defined to be the set of $C^{\infty}$ maps with domain $N$ and values in $S$. We shall call this diffeology the canonical diffeology on the $C^{\infty}$ manifold $S$.

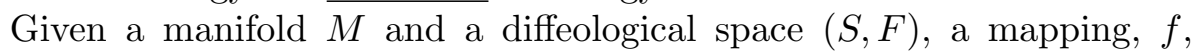
from a subset $C \subseteq M$ to $S$ is called smooth, when there exists an open neighborhood, $U$, of $C$ and a smooth extension of $f$ to $U, \tilde{f}: U \mapsto S$.

Given any collection of diffeological structures on a set $S, F_{i}$, we have that $\cap F_{i}$ is a diffeological structure, thus any assignment of functions, $G_{S}(U) \subset H_{\text {omet }}(U, S), U \subset E, E$ a Hausdorff locally convex topological vector space, generates a diffeology; namely, the smallest or finest diffeology containing the $G_{S}(U)$. For the diffeology so generated we shall call $G_{S}(U)$ a system of generators.

A useful notion for diffeological structures is that of the pull-back: given a diffeological stucture on a set $T,(T, G)$, and a function, $f: S \mapsto T$, define $f^{\star} G(U)=\left\{g \in H_{\text {om }}\right.$ sets $\left.(U, S): f \circ g \in G(U)\right\}$. It is straightforward to verify that $f^{\star} G$ is a diffeological stucture on $S$.

Given a subset $S_{1} \subset S_{2}$, where $\left(S_{2}, F_{2}\right)$ is a diffeological space, there is a diffeological structure induced on $S_{1}$ by $F_{1}(C)=\left\{f \in F_{2}(C): f(C) \subset S_{1}\right\}$. 
Note that $F_{1}=i^{\star}\left(F_{2}\right)$, where $i: S_{1} \mapsto S_{2}$ is the canonical inclusion. In the rest of this paper when we consider a subset of a diffeological space as a diffeological space it will be with the above described structure unless there is an explicit mention to the contrary. When $C$ is an open subset of a graded Hausdorff locally convex topologoical vector space we shall call $f$ of $F_{S}(C)$ a plot of the diffeological structure at a point $s=f(x), x \in C$.

Given diffeological spaces $\left(X_{1}, F_{1}\right)$ and $\left(X_{2}, F_{2}\right)$, and an open subset $U$ of an complete Hausdorff locally convex topological vector space $E$ a mapping $f: U \mapsto C^{\infty}\left(X_{1}, X_{2}\right)$ is smooth when $F: U \times X_{1} \mapsto X_{2}$, given by $F(u, x)=f(u)(x)$ is smooth. The finest diffeology on $C^{\infty}\left(X_{1}, X_{2}\right)$ admitting these functions as generating plots will be called the function space diffeology on $C^{\infty}\left(X_{1}, X_{2}\right)$ or the canonical diffeology on $C^{\infty}\left(X_{1}, X_{2}\right)$.

It follows from the definitions that

Proposition 2.1. Given compact $C^{\infty}$ manifolds $M$ and $N$ a function $f: N \mapsto C^{\infty}\left(M, R^{n}\right)$ is smooth for the $C^{\infty}$ topology on $C^{\infty}\left(M, R^{n}\right)$ if and only if the induced map $\tilde{f}: N \times M \mapsto R^{n}$ is $C^{\infty}$.

Corollary 2.1. Given a compact $C^{\infty}$ manifold $M$ and an open subset $U \subset R^{n}$ a function $f: U \mapsto D i f f^{\infty}(M)$ is smooth for the $C^{\infty}$ topology on Dif $f^{\infty}(M)$ if and only if the induced map $\tilde{f}: U \times M \mapsto M$ is $C^{\infty}$.

DEFinition 2.1. We shall call a diffeological structure lattice or $L$ type when given two plots $f: M_{1} \mapsto S, g: M_{2} \mapsto S$ at a point $t=f(x)=g(y) \in S$ there always exists a third plot through which the germs of $f$ at $x$ and $g$ at $y$ factor; that is, there exists a plot $h: N \mapsto S$ such that $f=h \circ \phi, g=h \circ \gamma$, where $\phi: \tilde{M}_{1} \mapsto N, \gamma: \tilde{M}_{2} \mapsto N$ are smooth functions such that $\phi(x)=\gamma(y)$, where $\tilde{M}_{i} \subset M_{i}$ is a neighborhood of $x$ (resp. $y$ ).

Suppose that $(S, F)$ is a diffeological space of $L-$ type; consider the equivalence relation generated between germs of one dimensional plots at $s \in S$ as follows: let $f$ and $g$ be one dimensional plots at $s$ from domains open intervals $C_{1}$ and $C_{2}$ containing 0 , we write $f_{0} \equiv g_{0}$, when $f(0)=g(0)=s$ and there exists a plot $\mathrm{k}: \mathrm{U} \mapsto S$ through which the germs of $f$ and $g$ factor at 0 and we have that $D_{0}(h \circ f)=D_{0}(h \circ g)$, where $h$ is any smooth real valued function defined on $k(U)$. The equivalence classes will be called tangent vectors at $s$, we shall designate the set of tangents at $s$ by $T_{s} S$.

It is immediate that a smooth map $f: S \mapsto W$ defines a function $T f$ : $T_{s} S \mapsto T_{f(s)} W$.

When $M$ is a manifold modelled on a locally convex topological vector space, this definition is equivalent to the classical one for the canonical diffeology associated to the manifold structure. Given a plot $f: U \mapsto S$, we use $T f: T U \mapsto T S$ to define plots on $T S$ and thus a diffeology on $T S$, in what follows $T S$ will be considered as a diffeological space with the finest diffeology admitting such $T f^{\prime} s$ as plots. 
Given diffeological spaces $\left(X_{1}, F_{1}\right)$ and $\left(X_{2}, F_{2}\right)$ the Cartesian product diffeology $F_{1} \times F_{2}$ on $X_{1} \times X_{2}$ is defined by $f \in F_{1} \times F_{2}$ if and only if $p r_{1} \circ f \in F_{1}$ and $p r_{2} \circ f \in F_{2}$. The cartesian product when considered as a diffeological space will be considered to have this cartesian product diffeology unless there is an explicit mention to the contrary.

A group $G$ with a diffeology $\mathcal{F}$ on its underlyling set will be called a diffeological group when multiplication and inversion define smooth maps $G \times G \mapsto G$ and $G \mapsto G$.

One readily verifies that each diffeological group is of $L-t y p e$.

In a similar vein we define other diffeological algebraic structures.

Given a plot at the identity, $e \in G, f: U \mapsto G, x \in U, U \subset E, E$ a locally convex topological vector space, $U$ open, define $D f(x ; \alpha)=\left[f(x+t \alpha]_{t=0}\right.$, $x \in U$.

In [7] we proved

Proposition 2.2. If $G$ is a diffeological group, then $T_{e} G$ is a diffeological vector space.

REMARK 2.1. Let $\{G, \mathcal{G}\}$ be a diffeological group and $N$ a normal subgroup of $G$, then one has that $G / N$ is a diffeological group, where the quotient diffeology on $G / N$ is the diffeology generated by the sets of functions $\{p \circ g, p: G \mapsto G / N$ canonical map, $g \in \mathcal{G}(U), U$ an open subset of a complete Hausdorff locally convex topological vector space\}.

We shall call a collection of plots $\mathcal{S}$ cofinal for a diffeological space $\{X, \mathcal{F}\}$ when every plot of $X$, factors germwise smoothly through a map of $\mathcal{S}$; that is, given a plot of $X, u: U \mapsto X$ and $u\left(x_{0}\right)=y_{0} \exists$ an open neighborhood of $x_{0}$ in $U, U_{0}$ and a smooth map $\phi: V_{0} \mapsto X \in \mathcal{S}$ such that $u \mid U_{0}=\phi \circ v$, where $v: U_{0} \mapsto V_{0}$ is smooth.

Definition 2.2. A diffeological group $G$ will be called a diffeological Lie group when the tangent space at the identity, $T_{e} G$, is a diffeological vector space which

(i) admits for every non zero $\alpha \in T_{e} G$ a smooth real valued linear map $T: T_{e} G \mapsto R$ such that $T(\alpha) \neq 0$, and

(ii) the linear plots of $T_{e} G$ are cofinal.

EXAMPLE 2.1. A Lie group, $G$ modeled on a $C^{\infty}$ super manifold (possibly infinite dimensional) admits the structure of a diffeological Lie group with the canonical diffeology.

TheOREM 2.1. Let $G$ be a diffeological Lie group, then $T_{e} G$ is a diffeological vector space which admits the structure of a Lie algebra such that the bracket operation defines a smooth linear map $\nabla_{X}: T_{e} G \mapsto T_{e} G$, where $\nabla_{X}(Y)=[X, Y]$. 
Let's recall a diffeological Lie group $G$ is called regular when the logarithmic derivative $f^{\prime}(t) f(t)^{-1}$ defines a diffeological isomorphism from the diffeological set of smooth mappings from the unit interval into $G$ which map zero to the identity, $e \in G, C_{0}^{\infty}(I, G)$, to the diffeological set of smooth mappings from the unit interval into $T_{e} G, \chi: C_{0}^{\infty}(I, G) \mapsto C^{\infty}\left(I, T_{e} G\right)$. Note that a map from the unit interval into a diffeological space is called smooth when there exists an open interval containing the unit interval to which the map can be smoothly extended.

It is straightforward to verify

Proposition 2.3. Given two regular diffeological Lie groups $G$ and $H$, and a smooth homorphism $F: G \mapsto H$, then $\operatorname{Hom}(I, d F) \circ \chi=\chi \circ \operatorname{Hom}(I, F)$

THEOREM 2.2. Let $G$ be a regular diffeological Lie group, then there exists a smooth function $\exp : T_{e} G \mapsto G$ such that $\exp ((t+s))=\exp (t \xi) \times$ $\exp (s \xi)$ and $D_{t}[\exp (t \xi)]=R_{\exp (t \xi)}(\xi)$.

COROLlary 2.2. If $G$ is a regular diffeological Lie group, then $T_{e} G$ admits the structure of a diffeological Lie algebra.

Definition 2.3. A diffeological vector space $E$ will be called integral when there exists a smooth linear map $\int: C^{\infty}(I, E) \mapsto E$, such that given any smooth real valued linear function $H: E \mapsto R$, we have $H\left(\int(f)\right)=$ $\int_{I} H(f(t)) d t$ and such that given any $v \in E$ we have $\int(f(t) v)=\left(\int_{I} f(t) d t\right) v$. We shall sometimes for the sake of clarity use the notation $\left.\int f(t)\right) d t$ especially when $\mathrm{f}$ takes its values in a function space.

Given an integral diffeological vector space, $E, f \in C^{\infty}(I, E), t \in I$ define $\int^{t}(f)=\int(f(t s) t) d s$

A subspace, $K$, of an integral diffeological vector space, $E$, will be called closed when $\int\left(C^{\infty}(I, K)\right) \subseteq K$.

Definition 2.4. Let $K\left\{x_{1}, \ldots, x_{n+2}\right\}$ be the free associative algebra over the field $\mathrm{K}$ generated by $x_{1}, \ldots, x_{n+2}, K\left\{x_{1}, \ldots, x_{n+2}\right\}$ is isomomorphic to the tensor algebra generated by an $n+2$ dimensional vector space.

Definition 2.5. Let $\mathcal{L}$ be an integral diffeological Lie algebra such that the linear plots of $\mathcal{L}$ are cofinal. A closed subalgebra, $\mathcal{H}$, will be called strongly integrable when

(i) $\int^{s}$ defines a smooth map $\int^{s}: C^{\infty}(I, \mathcal{L}) \mapsto C^{\infty}(I, \mathcal{L})$, and

(ii) when for each sequence $a_{i}(t) \in C^{\infty}(I, \mathcal{H}), \quad i=1, \ldots, n ; g(t) \in$ $C^{\infty}(I, \mathcal{H})$ and $P\left(x_{1}, \ldots, x_{n+2}\right) \in K\left\{x_{1}, \ldots, x_{n+2}\right\}$ the non homogeneous linear differential equation, $(\star) y^{\prime}=g(t)+P\left(a d_{a_{1}}(t), \ldots, a d_{a_{n}}\right.$ $\left.(y), \int^{s}\right)$ admits an unique smooth flow, $\Phi(a(t), s, l)$ such that $D_{s}$ $\Phi(a(t), s, l, g(s))=g(s)+[a(s), \Phi(a(t), s, l)], \Phi(a(t), 0, l)=l$ and such that $\Phi$ defines a smooth map

(iii) $\Phi: C^{\infty}(I, \mathcal{H}) \times I \times \mathcal{H} \times C^{\infty}(I, \mathcal{H}) \mapsto \mathcal{H}$, such that 
(iv) $\Phi(a(t), s, ; g): \mathcal{H} \mapsto \mathcal{H}$ is a diffeomorphism which induces a diffeomorphism

$$
\Phi: C^{\infty}(\mathrm{I}, \mathcal{I}) \mapsto C^{\infty}(\mathrm{I}, \mathcal{I})
$$

where $\Phi(f)(t)=\Phi(a, t, f(t))$

EXAMPLE 2.2. Let $M$ be a compact $C^{\infty}$ manifold and let $\mathcal{X}(M)$ be the Lie algebra of $C^{\infty}$ vector fields on $M$.

It results from the classical theory of differential equations on a compact manifold that the canonical diffeology associated to the $C^{\infty}$ topology on $\mathcal{X}(M)$ renders a closed sub algebra $\mathcal{H} \subseteq \mathcal{X}(M)$ a strongly integrable Lie algebra.

We proved in [7]:

THEOREM 2.3. Let $G$ be a simply connected, regular, diffeological Lie group with canonical diffeomorphism $\chi: C_{0}^{\infty}(I, G) \mapsto C^{\infty}\left(I, T_{e} G\right)$ and suppose that $H$ is a connected normal subgroup of $G$ such that there exists an integrable Lie ideal, $\mathcal{H} \subseteq T_{e} H \subseteq T_{e} G=\mathcal{G}$ with a diffeological vector space complement, $\mathcal{K}$ in $\mathcal{G}$, satisfying

(i) $\chi^{-1}\left(C^{\infty}(I, \mathcal{H})\right) \subseteq C_{0}^{\infty}(I, H)$;

(ii) given $h \in H$ suppose there exists a smooth path $f:[0,1] \mapsto H$ such that $f(0)=e, f(1)=h$, and $\chi(f) \in C^{\infty}(I, \mathcal{H})$;

(iii) given any $k \neq 0 \in \mathcal{G} / \mathcal{H}$, suppose there exists a smooth Lie algebra homomorpism $\phi: \mathcal{G} / \mathcal{H} \mapsto \mathcal{S}$, where $\mathcal{S}$ is the Lie algebra of a regular diffeological Lie group $S$ such that $\phi(k) \neq 0$.

Then $H$ and $G / H$ are regular diffeological Lie groups with Lie algebra $\mathcal{H}$ (resp. $\mathcal{G} / \mathcal{H}$.

THEOREM 2.4. Suppose that $\mathcal{G}$ is a strongly integrable diffeological Lie algebra such that $\int[a, f(t)]=\left[a, \int f(t)\right]$, where $f(t) \in C^{\infty}(I, \mathcal{G})$ and $a \in \mathcal{G}$. Then $C^{\infty}(I, \mathcal{G})$ with its canonical diffeology is a regular diffeological Lie group with a group product given by

$$
\left.(\star)(u \cdot v)(s)=\chi^{-1}(\chi(u)(s))+\Phi(\chi(v), s, \chi(u)(s))\right) .
$$

Proof. $(\star)[7]$ provides $C^{\infty}(I, \mathcal{G})$ with a group strructure. Since $\chi$ is a diffeomorphism of diffeological spaces it follows that

$C^{\infty}(I, \mathcal{G})$ is a diffeological group. It's Lie algebra is again $C^{\infty}(I, \mathcal{G})$ with Lie product given by

$$
\star \star \quad\{f, g\}(t):=\left[f(t), \int^{t} g(s)\right]+\left[\int^{t} f(s), g(t)\right]
$$

To see that $C^{\infty}(I, \mathcal{G})$ is an integral Lie algebra, suppose given a smooth $F$ : $I \mapsto C^{\infty}(I, \mathcal{G})$, define $\int F \in C^{\infty}(I, \mathcal{G})$ by $\int F(s)=\int F(t)(s) d t$. From the definitions and utilizing the equality $\int\left(\int f(x, y) d x\right) d y=\int\left(\int f(x, y) d y\right) d x$ we obtain that this new $\int: C^{\infty}\left(I, C^{\infty}(I, \mathcal{G})\right) \mapsto C^{\infty}(I, \mathcal{G})$ determines an integral 
Lie algebra stucture on $C^{\infty}(I, \mathcal{G})$. The above integral structure on $C^{\infty}(I, \mathcal{G})$ together with the strong integrability of $\mathcal{G}$ ) implies the strong integrability of $C^{\infty}(I, \mathcal{G})$.

Definition 2.6. Given two smooth paths $f, g: I \mapsto L$ into a diffeological Lie algebra, L, we say that $f, g: I \mapsto L$ are Lie homotopic when there exists smooth maps from the square $V, W: I \times I \mapsto L$ such that $V(t, 0)=$ $f, V(t, 1)=g, W(0, s) \equiv W(1, s) \equiv 0, V_{s}-W_{t}=[V, W]$

It has been shown [7] that the subset $N$ of $C^{\infty}(I, \mathcal{G})$ consisting of paths Lie homotopic to 0 is a normal subgroup of $C^{\infty}(I, \mathcal{G})$. Let $\Omega=\{\gamma \in$ $\left.C^{\infty}(I, \mathcal{G}): \int_{0}^{1} \gamma(s) d s=0\right\}$, it follows from $\star \star$ of the proof of theorem 2.4 that $\Omega$ is a Lie ideal of $C^{\infty}(I, \mathcal{G})$.

From $[\mathbf{7}]$ we have

ThEOREM 2.5. Suppose that $\mathcal{G}$ is a strongly integrable integral Lie algebra, then there exists a simply connected regular diffeological Lie group $G$ such that $T_{e} G \cong \mathcal{G}$.

Under these conditions we can now formulate a version of Lie's second fundamental theorem:

THEOREM 2.6. Let $\mathcal{G}$ be the integral Lie algebra of a simply connected regular difffeological Lie group and suppose that $\mathcal{H} \subseteq \mathcal{G}$ is a closed strongly integrable Lie subalgebra of $\mathcal{G}$. Then there exists a connected subgroup $H \subseteq G$ such that $T_{e} H \simeq \mathcal{H}$.

Proof. Let $\rho: C^{\infty}(I, \mathcal{G}) \mapsto C^{\infty}(I, \mathcal{G}) / N=G$ be the canonical Lie homomorphism of groups. The the smooth Lie group homomorphism of $C^{\infty}(I, \mathcal{H})$ into $C^{\infty}(I, \mathcal{G})$ induced by the canonical smooth Lie algebra homomorphism of $\mathcal{H}$ into $\mathcal{G}$ composed with $\rho$ gives a map onto a subgroup $H$ of $G$ which satisfies the conditions of the theorem.

\section{A method of construction of non-trivial graded compact supermanifolds with non degenerate graded differential forms}

For the rest of this paper we suppose that $\Gamma$ is the finitely generated Grassmann algebra with generators: $\xi_{1} \ldots \xi_{N}$, and suppose that $C$ is the canonical basis of $\Gamma$ associated to system of generators $\left\{\xi_{i}\right\}$. Let $\mathcal{M}$ be the category whose objects are super manifolds modelled on the open subsets of the graded $\Gamma_{0}$ module $V=\Gamma_{0}^{n} \oplus \Gamma_{1}^{m}$ having $G^{\infty}$ smooth maps of $G^{\infty}$ smooth super manifolds as its morphisms. As in the classical case we may define the tangent bundle to a supermanifolds $M$ to obtain a smooth vector bundle

$$
\Pi_{M}: T M \mapsto M
$$

where a vector $\alpha \in T_{x} M$ is defined to be an equivalence classes of smooth curves through $\mathrm{x}, a(t)$, parametrized by an open interval containing 0 such 
that $a(0)=x$ with the equivalence relation $a \equiv a^{\prime}$ if and only if $D_{t=0}(f \circ$ $a)(t)=D_{t=0}\left(f \circ a^{\prime}\right)(t)$ for every germ of a smooth function $f: U \mapsto \Gamma$ defined on a neighborhood $U$ of $x \in M$.

This definition is equivalent to the choice of a vector in the modeling space $\alpha_{i} \in E_{i}, U_{i} \subset E_{i}$ and $U_{j} \subset E_{j}$, and $\phi_{i}: U_{i} \mapsto M$ models $M$ with the equivalence relation at $x \in \phi_{i}\left(U_{i}\right) \cap \phi_{j}\left(U_{j}\right)$ given by: $\left.\left(U_{i}, \alpha_{i}\right)\right) \sim\left(U_{j}, \alpha_{j}\right)$ if and only if $D\left(\phi_{i}^{-1} \circ \phi_{j}\right)\left(\phi_{j}^{-1}(x), \alpha_{j}\right)=\alpha_{i}$, which implies

Proposition 3.1. $T_{x} M \cong \Gamma_{0}^{n} \oplus \Gamma_{1}^{m}=V$.

Definition 3.1. A supermanifold will be called pre-graded when the tangent bundle can be reduced to the group $G$ of $\Gamma_{0^{-}}$automorphisms of $\Gamma_{0}^{n} \oplus \Gamma_{1}^{m}$ such that $g \in G \Leftrightarrow g\left(\Gamma_{0}^{n}\right)=\Gamma_{0}^{n}$ and $g\left(\Gamma_{1}^{m}\right)=\Gamma_{1}^{m}$.

Proposition 3.2. Given a pre-graded supermanifold $M$, the even (resp. odd) vectors $E_{x}\left(\right.$ resp. $\left.O_{x}\right) \subset T_{x} M$ form a sub vector space of $T_{x} M$.

A pre-graded supermanifold is said to be graded when there exists a system of charts $\mathcal{U}=\left\{U_{i}\right\}, \phi_{i}: U_{i} \mapsto M$ for its supermanifold structure such that $D_{x}\left(\phi_{i}^{-1} \circ \phi_{j}\right)(x) \in G, \forall x \in U_{i} \cap U_{j}$. It follows from the definitions that

Proposition 3.3. If $M$ is a graded supermanifold, then there exists a subbundle $E,($ resp.O $) \subset T M$ such that $\alpha \in E_{x}\left(\right.$ resp. $\left.O_{x}\right)$ is vector tangent to a curve $C$ through $x$ with $\phi_{i}^{-1}(C) \subset \Gamma_{0}^{n}\left(\right.$ resp. $\Gamma_{1}^{m}$ such that $T M=E+O$.

Define $\delta\left(\xi_{i}\right)\left(s, x_{0}\right) \in G^{\infty}\left(U, \Gamma_{0}^{n} \oplus \Gamma_{1}^{m}\right)$ by $\delta\left(\xi_{i}\right)\left(s, x_{0}\right)=x_{0}+\left[\left(s \xi_{i} \oplus \ldots \oplus 0\right) \oplus \ldots \oplus\left(0 \oplus \ldots \oplus s \xi_{i}, \ldots, 0\right) \oplus \ldots \oplus\left(0 \oplus \ldots \oplus s \xi_{i}\right)\right]$, for $|s|>0$ sufficiently small.

Now define

$$
\partial f / \partial \xi\left(x_{0}\right): G^{\infty}(U, \Gamma)_{i} \mapsto G^{\infty}(U, \Gamma)_{i+1}
$$

by $\partial f / \partial \xi\left(x_{0}\right)=\Delta_{\xi} \circ D_{s=0} f\left(\delta(\xi)\left(s, x_{0}\right)\right)$, we recall $\Delta_{\xi}$ : Given a system of generators $\left\{\xi_{i}\right\}$ of $\Gamma$ define a linear map $\Delta_{\xi_{i}}: \Gamma \mapsto \Gamma$ by $\Delta_{\xi_{i}}\left(\xi_{i_{1}} \ldots \xi_{k} \ldots \xi_{i_{n}}\right)=$ $\sum_{k=1}^{n} \delta_{i i_{k}}(-1)^{(k-1)}\left(\xi_{i_{1}} \ldots \xi_{i_{k-1}} \xi_{i_{k+1}} \ldots \xi_{i_{n}}\right)$, and $\Delta_{\xi_{j}}\left(1_{\Gamma}\right)=0$.

Given an open subset $U \subset \Gamma_{0}^{n} \oplus \Gamma_{1}^{m}$, we define a graded right $\Gamma$-module structure on $G^{\infty}(U, \Gamma)$ by setting $G^{\infty}(U, \Gamma)_{0}=G^{\infty}\left(U, \Gamma_{0}\right)$ and $G^{\infty}(U, \Gamma)_{1}=$ $G^{\infty}\left(U, \Gamma_{1}\right)$, and setting $(f \gamma)(x)=f(x) \gamma$. As in the classical case we identify a vector $\alpha_{i} \in E_{i}$ with the class of $G^{\infty}$ curves in the direction of that vector and write $\partial / \partial x_{i}\left(\operatorname{resp} . \partial / \partial \xi_{i}\right)$ for the vector in the direction of $\eta_{i}$ (resp. $\left.\delta\left(\xi_{i}\right)\left(1, x_{0}\right)\right)$.

For the rest of this section we shall assume that our manifolds are graded supermanifolds and therefore that the fibers of $\Pi_{M}: T M \mapsto M$ have a canonically assigned gradation. The associated vector bundle with fiber $\Gamma \otimes_{\Gamma_{0}} V$

$$
\Pi_{M \Gamma}: T_{\Gamma} M \mapsto M
$$

inherits a graded structure by the convention $\left|\gamma \otimes_{\Gamma_{0}} v\right|=|\gamma|+|v|$. 
It is straightforward to prove that

Proposition 3.4. The $G^{\infty}$ smooth graded derivations of $G^{\infty}(U, \Gamma)$ are finite sums of the form

$$
\sum f_{i}(x, \xi) \partial / \partial \xi_{i}+g_{i}(x, \xi) \partial / \partial x_{i}+h_{i}(x, \xi) \partial / \partial \xi_{i}+k_{i}(x, \xi) \partial / \partial x_{i},
$$

where $(x, \xi) \in U \subset \Gamma_{0}^{n} \oplus \Gamma_{1}^{m}, f_{i}, k_{i} \in G^{\infty}(U, \Gamma)_{0}$, and $g_{i}, h_{i} \in G^{\infty}(U, \Gamma)_{1}$, designate by $\mathcal{D}(U)$ the space of derivations of $G^{\infty}(U, \Gamma)$, where $U \subset \Gamma_{0}^{n} \times \Gamma_{1}^{m}$, $x \in R^{n}$.

$\mathcal{D}(U)$ can be given the structure of a left $G^{\infty}(U, \Gamma)$ module by $(\gamma d)(f)$ $(x)=\gamma(x)(d(f)(x))$

Now let

$$
\Pi_{M}^{\star}: T_{\Gamma}^{\star} M \mapsto M
$$

be the associated bundle with fiber $\operatorname{Hom}_{\Gamma}\left(\Gamma \otimes_{\Gamma_{0}} V, \Gamma\right) \cong \operatorname{Hom}_{\Gamma_{0}}(V, \Gamma)$. Its fiber now is assigned a $Z_{2}$ grading by

$$
\begin{aligned}
\operatorname{Hom}_{\Gamma}\left(\Gamma \otimes_{\Gamma_{0}} V, \Gamma\right)_{0} & =\operatorname{Hom}_{\Gamma_{0}}\left(V_{i}, \Gamma_{i}\right) \text { and } \operatorname{Hom}_{\Gamma}\left(\Gamma \otimes_{\Gamma_{0}} V, \Gamma\right)_{1} \\
& =\operatorname{Hom}_{\Gamma_{0}}\left(V_{i}, \Gamma_{i+1}\right) .
\end{aligned}
$$

Given a trivializing chart at $x \in U \subset M$ for $\Pi_{M}: T M \mapsto M$

Suppose $\gamma_{i} \in \Gamma, \gamma_{0}=1, k=1, \ldots, N, j=1, \ldots, n$, is a basis of $\Gamma$ over $R$, then a basis of $T_{\Gamma x}^{\star} M$ over $R$ can be given by $d x_{j} \gamma_{i}, d \xi_{k} \gamma_{i}$ and $d x_{j}$, where $d \xi_{j} \in \operatorname{Hom}_{\Gamma_{0}}(V, \Gamma)$ are defined by $\left\{\partial / \partial x_{i} \mid d x_{j}\right\}=\delta_{j l}=\left\{\partial / \partial \xi_{i} \mid d \xi_{j}\right\}$ and $\{\partial /$ $\left.\partial \xi_{i} \mid d x_{j}\right\}=0=\left\{\partial / \partial x_{i} \mid d \xi_{j}\right\}$

Definition 3.2. A bilinear map $\omega: \mathcal{D}(U) \times \mathcal{D}(U) \mapsto G^{\infty}(U, \Gamma)$ is called a 2 - form when

$$
\begin{aligned}
\left\{X_{1}, f X_{2} \mid \omega\right\} & =(-1)^{\left|X_{1}\right||f|} f\left\{X_{1}, X_{2}, \omega\right\} \\
& =(-1)^{\left|X_{1}\right||f|}\left\{f X_{1}, X_{2}, \omega\right\}, f \in G^{\infty}(U, \Gamma) .
\end{aligned}
$$

We place a $Z_{2}$ gradation on 2 - forms such that $|\omega|=\left|\left\{X_{1}, X_{2} \mid \omega\right\}\right|+$ $\left|X_{1}\right|+\left|X_{2}\right|(\bmod 2)$

In the $Z_{2}$-graded case we now define wedge algebra of a graded vector space $V$ as follows: suppose that $R$ is the graded ideal generated by $\alpha \otimes \beta-$ $(-1)^{|\alpha||\beta|} \beta \otimes \alpha$ in the doubly graded tensor algebra

$$
\otimes^{\infty} V=\sum_{n=0}^{\infty} \otimes^{n} V,
$$

where $\alpha$ and $\beta$ are homogeneous elements of $V$.

Set $\bigwedge^{\infty} V=\otimes^{\infty} V / R \equiv \sum_{n=0}^{\infty} \bigwedge^{n} V$, where $\bigwedge^{n} V=V / R \cap \otimes^{n} V$,

On $\otimes^{\infty} V=\sum_{n=0}^{\infty} \otimes^{n} V^{6}$, a $Z_{2}$-gradation is placed on $\otimes^{n} V$ by the convention $\left|\alpha_{1} \otimes \cdots \otimes \alpha_{n}\right|=\left|\alpha_{1}\right|+\cdots+\left|\alpha_{n}\right|$. 
As in the classical case we may consider a smooth vector bundle $\wedge^{n} T^{\star}$ $M \mapsto M$ and smooth sections, $\omega$ of the linear bundle $\bigwedge^{n} T^{\star} M \mapsto M$, where $M$ is a supermanifold.

Rogers $[\mathbf{9}]$ p. 75 implies

Proposition 3.5. Given a trivializing chart $U$ of $\Pi_{M}^{\star} \mid \wedge^{2} T_{\Gamma}^{\star} U \mapsto U$, there is a 1-1 parity preserving correspondence between sections of $\Pi_{M}^{\star}\left|\Pi_{M}^{\star}\right|$ $\wedge^{2} T_{\Gamma}^{\star} U \mapsto U$ and 2-forms on $U$ determined by

$$
\begin{aligned}
\left\{X_{1}, X_{2} \mid \omega_{1} \wedge \omega_{2}\right\}= & (-1)^{\left|X_{2}\right|\left|\omega_{1}\right|}\left\{X_{1}, \mid \omega_{1}\right\}\left\{X_{2} \mid \omega_{2}\right\} \\
& -(-1)^{\left|\omega_{1}\right||| \omega_{2}|+| X_{1}|| \omega_{2} \mid}\left\{X_{1}, \mid \omega_{2}\right\}\left\{X_{2} \mid \omega_{1}\right\}
\end{aligned}
$$

REMARK 3.1. Derivations are are given the structure of left $G^{\infty}(U, \Gamma)$ modules and we give two forms the structure of right $G^{\infty}(U, \Gamma)$ module structure by the convention $\left\{X_{1}, X_{2} \mid \omega f\right\}=\left\{X_{1}, X_{2} \mid \omega\right\} f$.

f: Suppose that $A$ is an $n \times n$ upper triangular matrices of entries from $\left(\Gamma_{N}\right)_{0}$, with $1^{\prime} s$ down the diagonal; and suppose that $B$ is an $n \times m$ matrix with odd entries from $\left(\Gamma_{N}\right)_{1}$, and $I_{m}$ an $m \times m$ identity matrix.

The matrices of the form

$$
\left(\begin{array}{cc}
A & B \\
0 & I_{m}
\end{array}\right)
$$

form a connected nilpotent Lie group $G$ of dimension $2^{L-2} n(2 m+n-1)$.

Lemma 3.1. Let $H \subset G$ be a the subgroup whose entries are elements of $\left(\Gamma_{N}\right)_{0}$ and $\left(\Gamma_{N}\right)_{1}$ with integer coefficients of the canonical Grassmann generators $\xi_{i_{1}} \ldots \xi_{i_{k}} \ldots \xi_{i_{n}} ; i_{k} \leq i_{k+1}$. Then $H$ is a lattice subgroup of $G$.

Proof. $G$ is clearly nilpotent, we have only to establish that $G / H$ is compact. Firstly order the entries of the matrix lexicographically; that is, so that the entries are simply ordered by $(i, j) \prec(k, l)$ if and only if $i<k$ or $i=k$ and $j<l$.

We now perform an induction vis-a-vis this finite lexicographic simple ordering of the matrix entries.

For a real number $x$, designate by $\iota(x)$ the integral part of $x$. Given an element in $\Gamma, \gamma=\sum_{i=1}^{m} a_{j_{1}}, \ldots, j_{n_{i}} \xi_{j_{1}} \ldots \xi_{j_{k}} \ldots \xi_{j_{n_{i}}}$, designate by abuse of language $\iota(\gamma)=\sum_{i=1}^{m} \iota\left(a_{j_{1}}, \ldots, j_{n_{i}}\right) \xi_{j_{1}} \ldots \xi_{j_{k}} \ldots \xi_{j_{n_{i}}}$, where $j_{1}<\cdots<j_{k}<$ $\cdots<j_{n_{i}}$.

Now given a triangular matrix $\left(t_{i j}\right)=T \in G$ suppose that there exists a pair $k \leq l$ such that the entries of $T$ satisfy the property that $t_{i j}=\sum_{m=1}^{p}$ $a_{I_{1}, \ldots, i_{n_{i}}}^{(i j)} \xi_{I_{1}}, \ldots, \xi_{i_{k}}, \ldots, \xi_{i_{n_{m}}}$ with $\left|a_{I_{1}, \ldots, l_{n_{i}}}^{(i j)}\right| \leq 1$ when $(i j) \prec(k l)$.

Let $P_{k l}$ be the matrix which differs from the $n+m \times n+m$ identity matrix $I_{n+m}$ only in the $(\mathrm{k}, 1)$ th position where we suppose inserted $-\iota\left(t_{k l}\right)$ to obtain the matrix $P_{k l}$. 
Now observe that the matrix $\left(b_{i j}\right)=B=P_{k l} T$ has the property that $\left|b_{i j}\right| \leq 1$ for $(i j) \preceq(k, l)$, since $b_{k l}=t_{k l}-\iota\left(t_{k l}\right)$ and $b_{i j}=t_{i j}$ for $i j \prec k l$.

The above establishes that given any matrix $\left(t_{i j}\right)=T \in G$ there exists a matrix $P \in H$ such that $P T=B=\left(b_{i j}\right)$ has all of its entries $b_{i j}=\sum_{k} a_{i_{1}, \ldots, i_{k}}^{i j}$ $\xi_{i_{1}}, \ldots, \xi_{i_{k}}$ with real coefficients $\left|a_{i_{1}, \ldots, i_{k}}^{i j}\right| \leq 1$ with respect to th canonical basis $\xi_{i_{1}}, \ldots, \xi_{i_{k}}, \ldots, \xi_{i_{n}}$.

The above implies that $G / H$ is compact, and therefore that $H \subset G$ is a discrete lattice subgroup of the connected nilpotent Lie group $G$.

The homotopy sequence of the fibration $H \mapsto G \mapsto G / H$ implies that $\Pi_{1}(G / H) \cong H$.

Corollary 3.1. $G / H$ is a connected compact supermanifold modeled on $\left(\left(\Gamma_{N}\right)_{0}\right)^{n(n-1) / 2} \oplus\left(\left(\Gamma_{N}\right)_{1}\right)^{m n}$, such that $\Pi_{1}(G / H,[e]) \cong H$.

Example 3.1. (Odd Compact Graded Symplectic Manifold): Consider the Abelian super Lie group $G_{1} \subset G$ consisting of matrices of the form

$$
M=\left(\begin{array}{cc}
A & B \\
0 & 1
\end{array}\right)
$$

where $m=1$ and the first row of $A$ is a vector of the form $\alpha=\left(1, a_{12}, \ldots\right.$, $\left.a_{1 n+1}\right)$, where $a_{1 j} \in\left(\Gamma_{n}\right)_{0}$ and $B$ is of the form

$$
\left(\begin{array}{c}
b \\
0 \\
\vdots \\
0
\end{array}\right)
$$

where $b \in\left(\Gamma_{n}\right)_{1}$. Further, we suppose that eliminating the first row and column of $M, M$ becomes an $n \times n$ identity matrix.

The construction of Example 3.1 provides a torus $T=G_{1} / H \cap G_{1}$ of real dimension $2^{n-1}(n+1)$ modeled on $\left.\left(\left(\Gamma_{n}\right)_{0}\right)^{n} \oplus\left(\Gamma_{n}\right)_{1}\right)$.

Now suppose that $G_{1}$ is modeled at the identity by the trivializing chart $\left.\left.\left(a_{12}, \ldots, a_{1 n+1}\right), b\right) \mapsto\left(\left(\Gamma_{n}\right)_{0}\right)^{n} \oplus\left(\Gamma_{n}\right)_{1}\right)$. Let $d x_{j}, d \xi_{k} \in\left(T_{\Gamma}^{\star}\right)_{e} G$ be the elements determined by this trivialization at the identity. The right invariant Maurer Cartan form $\omega \in \Gamma\left(\bigwedge^{2} T_{\Gamma}^{\star} T\right)$ such that

$$
\Pi^{\star}(\omega(\text { identity }))=\sum_{i=1}^{n} d x_{i} d \xi_{i}
$$

determines the odd graded super-symplectic manifold structure on $T=$ $G_{1} / H \cap G_{1}$.

EXAMPLE 3.2. (even symplectic compact graded manifold): With the notation of the above example suppose that $\alpha=\left(1, a_{12}, \ldots, a_{12 s+1}\right)$ and suppose that $\Gamma$ is the Grassmanian algebra generated by $\xi_{1} \ldots \xi_{q}$. Now the construction of Example 3.1 gives a torus $T=G_{1} / H \cap G_{1}$ modeled on $\left(\left(\Gamma_{q}\right)_{0}\right)^{2 s} \oplus$ 
$\left.\left(\Gamma_{q}\right)_{1}\right)$ of real dimension $2^{q-1}(2 s+1)$. Consider the unique $\omega \in \Gamma\left(\bigwedge^{2} T_{\Gamma}^{\star} T\right)$ such that $\Pi^{\star}(\omega($ identity $))=2 \sum_{i=1}^{s} d x_{i} \wedge d x_{i+s}+\sum_{i=1}^{q} d \xi_{i} \wedge d \xi_{q-i}$.

The super symplectic structures of Examples 3.1 and 3.2 are nondegenerate in the sense that:

given any $X=\sum_{i=1}^{n} f_{i} \partial / \partial \xi_{i}+g_{i}(X, \Xi) \partial / \partial x_{i}+h_{i} \partial / \partial \xi_{i}+k_{i}(X, \Xi) f \partial / \partial x_{i}$, where $f_{i}, g_{i} \in \Gamma$ there exists $Y=\sum_{i=1}^{n} h_{i} \partial / \partial \xi_{i}+k_{i} \partial / \partial x_{i}$ such that $\omega(X, Y) \neq 0$

EXAMPLE 3.3. (compact graded contact super manifold)

With the notation of the above example suppose that $\alpha=\left(1, a_{12}, \ldots\right.$, $\left.a_{12 s+2}\right)$ and suppose as above that $\Gamma$ is the Grassmanian algebra generated by $\xi_{1} \ldots \xi_{q}$. Now the construction of Example 3.1 gives a torus $T=G_{1} / H \cap G_{1}$ modeled on $\left.\left(\left(\Gamma_{q}\right)_{0}\right)^{2 s+1} \oplus\left(\Gamma_{q}\right)_{1}\right)$ of real dimension $2^{q-1}(2 s+2)$. Consider the unique 1-form $\omega \in \Gamma\left(T_{\Gamma}^{\star} T\right)$ such that $\Pi^{\star}(\omega($ identity $))=2 \sum_{i=1}^{s}\left(x_{i} d x_{i+s}-\right.$ $\left.x_{s+i} d x_{i}\right)+\sum_{i=1}^{q} \xi_{i} d \xi_{q-i+1}$.

EXAMPLE 3.4. (graded compact odd contact super manifold) In Example 3.3 we suppose $\Gamma$ is generated by $\xi_{1} \ldots \xi_{n+1}$ and that $T=G / H$ is the torus modeled on $\left((\Gamma)_{0}\right)^{n} \oplus(\Gamma)_{1}$ of real dimension $2^{n+1}(n+1)$. Consider the unique 1 -form

$$
\omega \in \Gamma\left(T_{\Gamma}^{\star} T\right)
$$

such that $\Pi^{\star}(\omega($ identity $))=d \xi_{n+1}+\sum_{i=1}^{n}\left(x_{i} d \xi_{i}+\xi_{i} d x_{i}\right)$.

\section{Automorphisms of graded compact manifolds}

It is known [5] that

THEOREM 4.1. Suppose that $M$ is a compact $G^{\infty}$ manifold modeled on $\Gamma_{0}^{n} \oplus \Gamma_{1}^{m}$, where $\Gamma$ is the algebra generated by the finite sequence $\xi_{1}, \ldots, \xi_{k}, \ldots$, $\xi_{N}$. The tangent space $T_{x} M$ has canonically $\Gamma_{0}$ module structure such that $T M$ is a $G^{\infty}$ manifold and such that the canonical projection $T M \mapsto M$ is a $G^{\infty}$ bundle.

Let Dif $f^{\infty}(M)$ and Dif $f^{\infty}(M)$ be respectively the group of $C^{\infty}$ (resp. $\left.G^{\infty}\right)$ diffeomorphisms of $M, \operatorname{Diff}^{\infty}(M)$ and $\operatorname{Dif} f^{G^{\infty}}(M)$ are regular diffeological Lie groups with Lie algebras $\mathcal{X}$ and $\mathcal{D}$, where $\mathcal{X}$ represent the $C^{\infty}$ vector fields on $\mathrm{M}$ and $\mathcal{D}$ is the closed Lie subalgebra of $\mathcal{X}$ of $G^{\infty}$ vector fields on $M$ with the canonical $\Gamma_{0}$ module structure. Further $\mathcal{X}$ and $\mathcal{D}$ are strongly integrable Lie algebras in the sense of section 2 .

REMARK 4.1. The modeling of $\operatorname{Diff} f^{G^{\infty}}(M)$ is done by the restriction of the exponential map of a $G$-connection on $M$, $\exp _{G}: \mathcal{X} \mapsto D$ iff $f^{\infty}(M)$ to $\mathcal{D}$ and defines a $G^{\infty} \operatorname{map}\left(\exp _{G} \mid \mathcal{D}\right): \mathcal{D} \mapsto D i f f G^{\infty}(M)$, where $G \subset G L\left(\Gamma_{0}^{n} \oplus\right.$ $\left.\Gamma_{1}^{m}\right)=G L\left(2^{N-1}(m+n), R\right)$, is the subgroup of of $\Gamma_{0}$ automorphisms of $\Gamma_{0}^{n} \times \Gamma_{1}^{m}$. 
Diff $G^{\infty}(M)$ is the group of automorphisms of this $G$ structure. The manifold structure on Diff $f^{\infty}(M)$ is the canonical closed submanifold structure induced by the $C^{\infty}$ manifold structure on Dif $f^{\infty}(M)$.

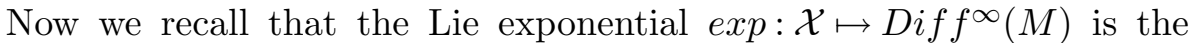
unique solution of the differential equation on the compact manifold $M$ determined by

$$
(\star \star) d / d t\left(\exp (t, X)=X(\exp (t, X)), \exp (0, X)=i d_{M} \in D i f f^{\infty}(M) .\right.
$$

This differential equation restricted to $\mathcal{D}$ determines a $G^{\infty}$ differential equation on the compact $G^{\infty}$ manifold $M$ so that exp detemines locally a $G^{\infty}$ mapping exp $: \mathcal{D} \mapsto \operatorname{Diff}^{G^{\infty}}(M)$.

Corollary 4.1. Given $X \in \mathcal{D}$ the Lie derivative defines a $\Gamma_{0} p$ contravariant (q covariant) homorphism $L_{X}: \Gamma\left(\wedge^{p}\left(T_{\Gamma}^{\star} M \otimes_{\Gamma_{0}} \otimes_{\Gamma_{0}}^{q} T_{\Gamma} M\right)\right) \mapsto$ $\Gamma\left(\wedge^{p}\left(T_{\Gamma}^{\star} M \otimes_{\Gamma_{0}} \otimes_{\Gamma_{0}}^{q} T_{\Gamma} M\right)\right)$, where $\Gamma\left(\wedge^{p}\left(T_{\Gamma}^{\star} M\right) \otimes_{\Gamma_{0}} \otimes_{\Gamma_{0}}^{q} T_{\Gamma} M\right)$ represents the smooth sections of the bundle

$$
\Pi_{M}: \wedge^{p} T_{\Gamma}^{\star} M \otimes_{\Gamma_{0}} \otimes_{\Gamma_{0}}^{q} T_{\Gamma} M \mapsto M
$$

and induces a $G^{\infty} \operatorname{map}(X, \omega) \mapsto L_{X}(\omega), \mathcal{D} \times \Gamma\left(\wedge^{p}\left(T_{\Gamma}^{\star} M\right) \otimes_{\Gamma_{0}} \otimes_{\Gamma_{0}}^{q} T_{\Gamma} M\right) \mapsto$ $\Gamma\left(\wedge^{p}\left(T_{\Gamma}^{\star} M\right) \otimes_{\Gamma_{0}} \otimes_{\Gamma_{0}}^{q} T_{\Gamma} M\right)$.

THEOREM 4.2. Suppose that $M$ is a compact $G^{\infty}$ manifold modeled on $\Gamma_{0}^{n} \oplus \Gamma_{1}^{m}$, where $\Gamma$ is the Grassmann algebra generated by the finite sequence $\xi_{1}, \ldots, \xi_{k}, \ldots, \xi_{N}$. Given $\omega \in \Gamma\left(\wedge^{p}\left(T_{\Gamma}^{\star} M\right) \otimes_{\Gamma_{0}} \otimes_{\Gamma_{0}}^{q} T_{\Gamma} M\right)$, Let $W=\{X \in \mathcal{D}$ : $\left.L_{X}(\omega)=0\right\}$.If $W$ is Lie sub algebra of $\mathcal{D}$, then $W$ is a strongly integrable Lie sub algebra of $\mathcal{D}$.

Idea of Proof: $W \subset \mathcal{D} \subset \mathcal{X}$ is a closed Lie sub algebra for the $C^{\infty}$ topology, and therefore the differential equation $(\star) y^{\prime}=g(t)+P\left(a d_{a_{1}}(t), \ldots\right.$, $\left.a d_{a_{n}}(y), \int^{s}\right)$ of definition 2.4 induces a smooth differential equation on the compact manifold $M$, the existence and uniqueness theorem for compact manifolds imply that $\star$ admits a solution in $W$ with the required properties.

\section{References}

[1] Borel, A. and Wallach, N., Continuous cohomology, discrete subgroups and representations of reductive groups, Ann. of Math. Stud. 94 Princeton University Press, Princeton, NJ, 1980.

[2] Cantarini, N. and Kac, V., Infinite-dimensional Primitive Linearly Compact Lie Superalgebras, Adv. in Math, (2006), 328-419.

[3] Iglesias, P., Thesis, L'Universite de Provence, 1985.

[4] Leslie, J., On the Lie Subgroups of Infinite Dimensional Lie Groups, Bull. of AMS Jan.(1987).

[5] Leslie, J., On a Super Lie Group Structure for the Group of $G^{\infty}$ Diffeomorphisms of a compact $G^{\infty}$ supermanifold Geometry \& Physics. (1997).

[6] Leslie, J., Lie's Third Theorem in Supergeometry, Algebras, Groups, and Geometries. 14:4(1997), 359-406. 
[7] Leslie, J., On a Diffeological Group Realization of Certain Generalized Symmetrizable Kac-Moody Lie Algebras, Journal of Lie Theory 13 (2003), 427-442.

[8] Milnor, J., Remarks on Infinite Dimensional Lie Groups, Proc. of Summer School on Quantum Gravity (1983).

[9] Rogers, A., Supermanifolds Theory and Applications, World Scientific (2008).

[10] Singer, I. M. and Sternberg, S., On the infinite groups of Lie and Cartan, I. Ann Inst, Fourier (Grenoble) 15 (1965), 1-114.

[11] Souriau, J. M., in "Feuilletages et Quantification geometrique", Travaux en Cours, Hermann, 1984, 365-398. 
